\title{
The Usefulness of STEAP Proteins in Prostate Cancer Clinical Practice
}

\author{
Sandra M Rocha ${ }^{1}$ • Jorge Barroca-Ferreira ${ }^{1,2}$ - Luís A Passarinhat,2,3 • \\ Sílvia Socorro ${ }^{1}$ • Cláudio J Maia ${ }^{1}$ \\ ${ }^{1}$ CICS-UBI - Centro de Investigação em Ciências da Saúde, Universidade da Beira Interior, \\ Covilhã, Portugal; ${ }^{2} \mathrm{UCIBIO}$ - Unidade de Ciências Biomoleculares Aplicadas, \\ Departamento de Química, Faculdade de Ciências e Tecnologia, Universidade Nova de \\ Lisboa, Caparica, Portugal; ${ }^{3}$ Laboratório de Fármaco-Toxicologia - UBIMedical, \\ Universidade da Beira Interior, Covilhã, Portugal.
}

Author for correspondence: Cláudio J Maia, Centro de Investigação em Ciências da Saúde, Universidade da Beira Interior, Covilhã, Portugal. E-mail: cmaia@fcsaude.ubi.pt Doi: https://doi.org/10.36255/exonpublications.prostatecancer.steap.2021

\begin{abstract}
Prostate cancer is a multifactorial disease and the second most common cancer diagnosed in men worldwide. The six transmembrane epithelial antigen of prostate (STEAP) proteins seem to be involved in prostate tumorigenesis. The STEAP proteins are differentially expressed in prostate cancer cells, and survival analysis reveal that prostate cancer patients with high levels of STEAPl have poor survival outcomes. In contrast, high expression of STEAP4 offers a better prognosis. This chapter provides an overview of the role of STEAP proteins in prostate cancer. The structure, biological functions, and the potential prognostic significance of each of the four members of the STEAP family in prostate cancer are discussed.
\end{abstract}

Keywords: biomarker; prognosis; prostate cancer; STEAP proteins; survival

In: Prostate Cancer. Bott SRJ, Ng KL (Editors). Exon Publications, Brisbane, Australia. ISBN: 978-0-6450017-5-4; Doi: https://doi.org/10.36255/exonpublications.prostatecancer.2021

Copyright: The Authors.

License: This open access article is licenced under Creative Commons Attribution-NonCommercial 4.0 International (CC BY-NC 4.0) https://creativecommons.org/licenses/by-nc/4.0/ 


\section{INTRODUCTION}

Prostate cancer is the second most diagnosed cancer and the sixth leading cause of cancer-related death in men in the Western world. Each year, 1.6 million men are diagnosed with this neoplasia, and 366,000 men die of prostate cancer (1). In 2040, 2.3 million new cases and 740, 000 prostate cancer-related deaths are estimated to occur worldwide due to the growth and aging of population (1). Endogenous (age, family history, ethnicity, hormones, and oxidative stress) and exogenous (dietary factors, physical inactivity, obesity, environmental factors, occupation, smoking) risk factors contribute to the risk of developing prostate cancer. However, older age, black race, and a family history of the disease are the best-established risk factors for prostate cancer (2).

The main biomarker used in clinical practice for prostate cancer screening is the serum level of prostate-specific antigen (PSA). However, several factors may affect PSA levels resulting in a considerable number of false-positives (3). The low specificity of PSA in the diagnosis of prostate cancer is a clinical problem. There is an urgent need to identify new biomarkers for early detection of the disease, and to improve patients' stratification and better define targeted therapies and clinical management of prostate cancer.

The human six-transmembrane epithelial antigen of prostate (STEAP) family of proteins comprises four members, namely STEAP1 to STEAP4, which are involved in numerous biological processes including the control of cell proliferation and apoptosis (4), oxidative stress (5) and molecular trafficking in exocytic and endocytic pathways (6). Cumulative evidence has pointed out STEAP family members as putative biomarkers, as well as therapeutic targets, in several types of human cancers, particularly in prostate cancer (7-11). However, the clinical significance of the expression of STEAP proteins for prostate cancer development is still scarce, and further analysis is required to ascertain their usefulness as prognostic biomarkers. This chapter first provides an overview of the structure and biological functions of STEAP proteins, followed by a discussion on their role in prostate cancer. Their putative role in tumorigenesis and prognosis of prostate cancer, based on datasets retrieved from the cBioPortal (12) and CANCERTOOL (13) public databases are presented.

\section{STRUCTURE AND ROLE OF STEAP PROTEINS}

All STEAPs have a characteristic six-transmembrane helix with intracellular Nand C-terminal domains, with a homologous architecture to ion-channels and/or transporter proteins $(6,8,14,15)$. The C-terminal domain is similar to the transmembrane domain (TMD) of the yeast ferric reductase (FRE) family of b-type cytochrome metalloreductases, whereas the N-terminal is comparable to the archaeal and bacterial $\mathrm{F}_{420}$ :NADPH-oxidoreductase (FNO)-binding proteins and to human NADPH-oxidoreductase domains (OxRD) $(6,8,14,15)$. The FNO-like domain reinforces the importance of STEAPs in the uptake and reduction of molecular oxygen and chelation of metal ions $\mathrm{Fe}^{3+}$ and $\mathrm{Cu}^{2+}$, and the involvement of these proteins in transmembrane-electron transport through the intracellular binding of NAD and FMN nucleotides to a conserved single heme-binding 


\section{STEAP1}

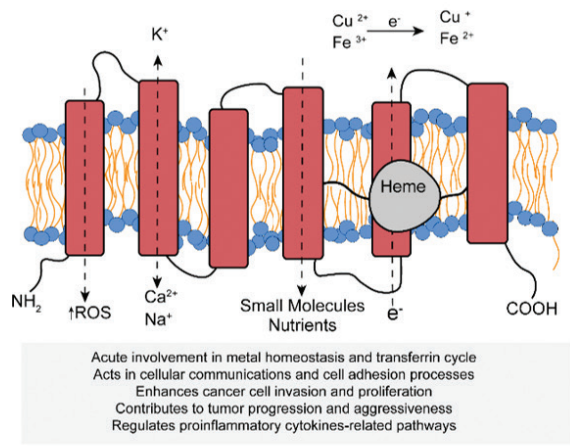

STEAP3

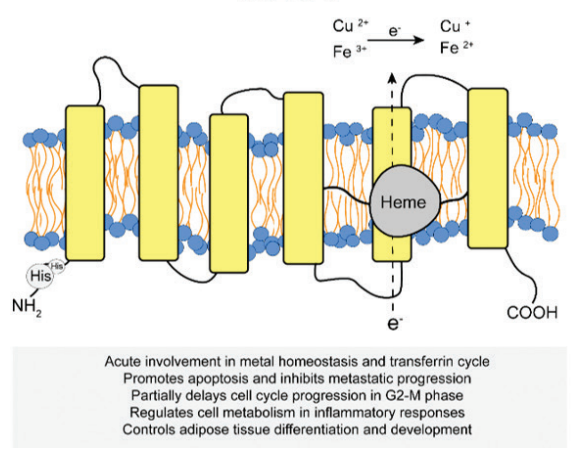

STEAP2

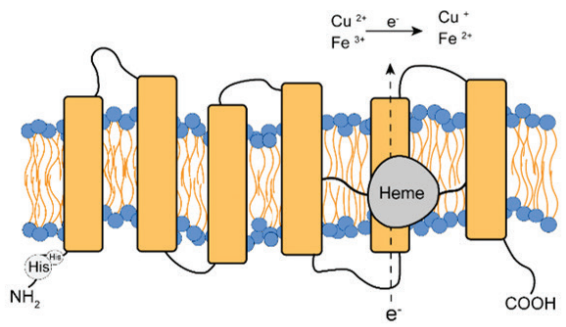

Acute involvement in metal homeostasis and transferrin cycle Acute involvement in metal homeostasis and transferrin cycle
Acts as cell-surface ferrirudectase and controls molecular trafficking Enhances cancer cell invasion and proliferation Regulates in endo-and exocytic pathways
Partially arrests cell cycle progression in G0-G1 phase

\section{STEAP4}

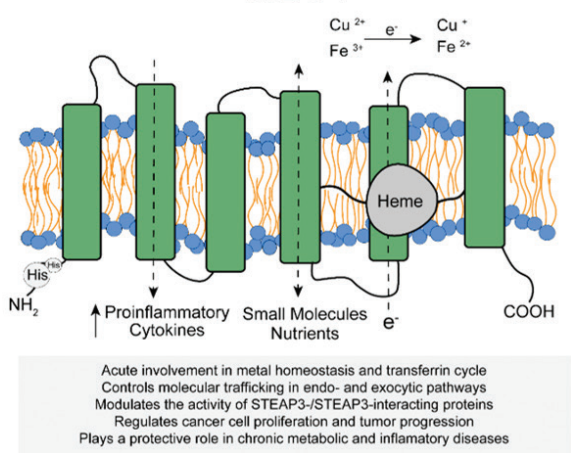

Figure 1. Schematic structure of STEAP family members. Representation of STEAP1, STEAP2, STEAP3 and STEAP4 proteins and their main cellular and biological functions.

histidine residue and a flavin-binding site $(6,14,15)$. Also, STEAPs share a YXX $\varnothing$ consensus sequence responsible for targeting transmembrane proteins to lysosomes and endosomes, and the Rossmannfold (GxGxxG/A motif) that binds NAD and FMN coenzymes (6). Besides maintaining cellular metal homeostasis, STEAPs are implicated in several biological processes, such as oxidative stress response, inflammation, metabolism, invasion, proliferation, growth, and apoptosis (Figure 1) (8).

\section{STEAP1}

The STEAP1 gene is located on chromosome 7q21.13 close to STEAP1B, STEAP2, and STEAP4, in a region that contains a cluster of genes predicted to encode transmembrane proteins. It encodes an mRNA of $1.3 \mathrm{~kb}$ that is translated into a mature protein of 339 amino acids (UniProt ID: Q9UHE8, $39.851 \mathrm{kDa}$ ). The STEAP1 protein is composed of six-transmembrane domains with cytosolic C- and N-terminals connected by three extra- and two intracellular loops preferentially 
located at tight and gap junctions (16). Unlike other STEAPs, STEAPl lacks the N-terminal NADPH-oxidoreductase, the FNO-like domain, and the Rossmann fold $(8,14)$. Nevertheless, STEAPl partially co-localizes with transferrin (Tf), transferrin receptor (TfR), and lysosomes or endosomes, suggesting an involvement in metal homeostasis $(6,17)$. A recent study indicated that dithionitereduced purified STEAPl is capable of reducing metal-ion complexes and molecular oxygen through a conserved heme-binding site (18). Furthermore, the amino acid sequence, the transmembrane topology, and cellular membrane localization of STEAPl indicate that it may act as an ion channel or transporter, modulating the concentration of small molecules, ions and nutrients, and also releasing soluble cytokines and chemokines (19). These features pointed out the role of STEAPl in cellular communication and in cell adhesion processes $(19,20)$. However, the recent cryogenic electron microscopy (cryo-EM) structure of STEAPl transmembrane domain bound to an antigen-binding fragment of an antibody (mAb 120.545) revealed a trimeric arrangement quite distinct from typical ion channels or transporters (21). The proposed unit indicated that STEAPl is a functional reductase in heterodimers complexes with other STEAP paralogs with a domain-swapped architecture with the intracellular OxRD positioned beneath the TMD of the adjacent promoter $(21,22)$. This arrangement supports a model in which the heme-binding site recruit and orient intracellular electron-donating substrates bound to an adjacent STEAP2-4 subunit, enabling transmembrane electron transport and the reduction of extracellular metal-ion complexes $(18,21)$. These structural features highlight the usefulness of STEAPl as a promising therapeutic target and biomarker for cancer and encouraged the development of strategies targeting STEAP1. In vitro and in vivo studies revealed mobilized dendritic cells and immunogenic STEAP1-derived peptides suitable for recognition by cytotoxic T lymphocytes for further development of anti-cancer vaccines (23). Humanized variant of anti-STEAPl monoclonal antibody (mAb 120.545) is currently used in prostate cancer clinical trials as an antibody-drug conjugate (DSTP3086S) (24), and as a radiolabeled antibody (89Zr-DFO-MSTP2109A) for PET imaging $(24,25)$. In addition, several studies exploring the role of STEAPl in cancer cells showed that its overexpression inhibits apoptosis, enhances cell proliferation and invasion, and induces epithelial to mesenchymal transition, ultimately contributing to tumor progression and aggressiveness (4, 5, 26-30).

\section{STEAP2}

The STEAP2 gene is located on chromosome 7q21.13, close to STEAP1 and STEAP4, and encodes an mRNA of $2.2 \mathrm{~kb}$, which generates a protein with 490 amino acids (UniProt ID: Q8NFT2, $52.052 \mathrm{kDa}$ ). The protein shuttles between the plasma membrane and Golgi complex in prostate epithelial cells (31). Its association with the trans-Golgi network and early endosomes suggests the involvement of STEAP2 in endo- and exocytic pathways, acting as a regulator of processing, secreting and sorting mechanisms of prostate-specific proteins, or as a receptor for endo- or exogenous ligands $(31,32)$. Moreover, as STEAP2 colocalizes with $\mathrm{Tf}$ and $\mathrm{TfR}$, the protein contributes to iron and copper reduction, and plays a role in the endosomal Tf cycle of erythroid cells, then controlling the 
molecular trafficking and availability of metals $(6,17)$. STEAP2 regulates several genes involved in cell cycle progression. Downregulation of STEAP2 results in a partial cell cycle arrest in the G0/Gl phase, suppression of prostate cancer cell proliferation, invasiveness, and metastatic potential (33). In contrast, STEAP2 overexpression increases the migration and invasion abilities of prostate cancer cells, and is associated with advanced cancer stage and histologic grading $(34,35)$. Although STEAP2 presents proliferative features and acts as a pro-survival factor, more studies exploring the molecular and signaling pathways underlying prostate cancer are required.

\section{STEAP3}

The STEAP3 gene is located on chromosome 2q14.2 and encodes an mRNA of $4.3 \mathrm{~kb}$, generating a protein with 488 amino acids (UniProt ID: Q658P3, 54.601 $\mathrm{kDa}$ ). The predicted structure of STEAP3 indicates six-transmembrane domains at C-terminal region, a cytosolic OxRD at the N-terminal counterpart and two conserved histidine residues (36). The crystal structure of cytoplasmic OxRD showed similarity with integral membrane cytochromes (37). The STEAP3 has unique structural and biochemical properties, revealing an FNO-like domain with a dimer interface and substrate binding sites, to direct electron transfer from the cytosol to a single b-type heme moiety predicted to be fixed within the TMD $(14,37)$. Furthermore, the N-terminal OxRD was found to dimerize, suggesting that STEAP3 is active as a homo- and/or heterodimer, and is found in cellular membranes to permit intercellular electron flow $(14,37)$. STEAP3 is mainly located in the plasma and endosomal membranes, and as a component of trans-Golgi network and endosomal-vesicular compartments (38). Also, STEAP3 is essential for the assembly of exosomes and vesicular proteins trafficking (39), and extracellular matrix organization (40). Moreover, the partial colocalization with Tf, TfR and divalent metal transporter 1 (DMT1), along with STEAP3 structural arrangement, suggest an important biological role of this protein in ferric compounds metabolism and erythroid transferrin cycle $(14,41)$. These functions were confirmed through the analysis of STEAP3 crystal structure, which postulated that the protein might function within a large complex with DMTl and Tf:TfR, ensuring the reduction and uptake of iron and copper at the cell surface (37). Several studies pointed out that STEAP3 is the major ferric reductase and deeply involved in regulating metal homeostasis in developing erythrocytes, macrophages, hepatocytes and endosomes (41). Therefore, tight regulation of intracellular iron is crucial to control cancer cell proliferation and apoptosis, and inhibiting the metastatic process (42). Furthermore, STEAP3 may be considered a tumor suppressor gene, acting as an intrinsic apoptosis factor. Studies showed that this protein suppresses the growth of human prostate cancer cells and could directly induce apoptosis through a caspase- 3 dependent pathway $(43,44)$. On the other hand, the increased expression of STEAP3 is related to the progression of prostate cancer in late stages of disease, ultimately contributing to metastization (45). Altogether, available data highlight the importance of STEAP3 as a potential tumor suppressor protein, a feature that contrasts with other STEAPs. 


\section{STEAP4}

The STEAP4 gene is located on chromosome 7q21.12 and encodes an mRNA of $4.5 \mathrm{~kb}$, and a protein composed of 459 amino acids (UniProt ID: Q687X5, 51.981 $\mathrm{kDa}$ ). STEAP4 is located at the plasma membrane, near the nuclear region, where it co-localizes with the Golgi complex, the trans-Golgi network and early endosomes (46). As with STEAP2 and STEAP3, the STEAP4 protein is involved in molecular trafficking either in endo- or exocytic pathways, and might be critical to cellular and systemic metal homeostasis $(6,17)$. The STEAP4 protein is also found dispersed in the cytoplasm within vesicular-tubular structures or reticular shapes associated with the estrogen receptor (ER), wherein it acquires its active conformation (46). Initially, it was predicted that STEAP4 was an integral sixtransmembrane metalloreductase composed of cytoplasmic N-terminal OxRD and a C-terminal TMD with six-membrane spanning $\alpha$-helices enveloping a single heme-binding site (14). However, the recently solved cryo-EM structure of human STEAP4 demonstrated an aligned inter-subunit trimeric NADPH-FAD-heme domain-swapped assembly containing both OxRD and six-helical TMD $(15,22)$. This arrangement facilitates the transport of intracellular electrons from NADPH through membrane-embedded FAD that flips to anchor itself in the inner-membrane region of the adjacent STEAP subunit, and heme co-factors to chelated metal-ion complexes at the extracellular membrane side $(14,15,22)$. Altogether, these biochemical and structural studies suggest that STEAP4 is a functional protein by establishing homo- or heterodimers with other STEAPs paralogs, which indicate that increased expression of STEAP4 could modulate the activity of STEAP3, and STEAP3-interacting proteins, such as NIX, MYT1, NIP3L, FAK-1, S100B, RHBDL4/RHBDD1 $(14,37,47)$. Moreover, these interactions suggest a link to metal homeostasis, apoptosis, differentiation, and cell cycle progression (47). The STEAP4 protein is involved in cellular responses to nutritional and inflammatory signals, particularly as glucose homeostasis regulator in adipocytes, besides being widely associated with cardiac malfunctions, hepatic and mitochondrial metabolic dysfunctions, skeletal system-related disorders, and also as a suppressor of pro-inflammatory cytokines (47). In addition to its role in cellular or systemic homeostasis, STEAP4 is associated with tumorigenesis. Considering its metal reductase activity, an overexpression of STEAP4 increases oxidative stress, contributing to increased mutational rates, proliferation and progression of prostate cancer cells (48). The effects of STEAP4 on prostate cancer growth and survival are due to the modulation and regulation of the expression and activity of focal adhesion kinase (FAK) and activating transcription factor (ATF4) through increased intracellular reactive oxygen species, which depend on the OxRD of STEAP4 $(48,49)$. It was recently demonstrated that ATF4-target genes promote prostate cancer cell survival and are upregulated in late stages of the disease (50). These considerations indicate a protective role of STEAP4 in inflammatory stress in chronic metabolic and inflammatory diseases. Besides, they pointed out an active role of STEAP4 in cancer cell proliferation and tumor progression, highlighting the importance of understanding its putative function in disease-related environment. 


\section{STEAP IN PROSTATE CANCER}

STEAP family members have been implicated in several human cancers, including prostate cancer. In non-neoplastic prostate, the expression of STEAP1 mRNA and protein are higher when compared to the other three family members (8). With the development of prostate cancer, the overall expression of STEAPs is dysregulated or reversed. STEAPl expression is highly increased in prostate cancer in comparison with non-malignant tissues (Table 1) $(16,27,28)$. Also, STEAPl staining intensity correlated with tumor grading, suggesting that it is associated with malignant transformation and tumor aggressiveness $(27,28)$. The aggressiveness and prognosis of prostate cancer is traditionally determined by Gleason score, and a recent study by Burnell et al. showed that STEAPl staining intensity in non-malignant tissue was weak, increasing slightly in Gleason 6 prostate cancer samples, and strongly from Gleason 7 onwards (7). Nowadays, public databases are widely used to corroborate basic research. The Prostate Adenocarcinoma dataset (51) from cBio Cancer Genomics Portal (https://cbioportal.org [accessed on December 2020]), was analyzed for STEAPs mRNA expression with a z-score threshold of \pm 1.8 . Of the 150 patients queried, STEAPl was overexpressed in $17 \%$, and underexpressed in $0.8 \%$ of patients. Bioinformatics analysis using the CANCERTOOL software (http://genomics.cicbiogune.es/ [accessed on December 2020]), which uses transcriptomics databases for the most prevalent types of cancers, demonstrated that increased expression of STEAP1 correlates with the onset of prostate cancer and development of metastatic disease (Figure 2).

\begin{tabular}{|c|c|c|c|}
\hline \multirow[t]{2}{*}{ TABLE 1} & \multicolumn{3}{|c|}{$\begin{array}{l}\text { Relative expression levels of STEAP family } \\
\text { members in human prostate cancer compared } \\
\text { with non-malignant tissue }\end{array}$} \\
\hline & & Expression pattern & Reference \\
\hline \multirow[t]{2}{*}{ STEAP1 } & mRNA & Increased & $(16,27,28)$ \\
\hline & Protein & & \\
\hline \multirow[t]{2}{*}{ STEAP2 } & mRNA & Increased & $(31,32)$ \\
\hline & Protein & & \\
\hline \multirow[t]{2}{*}{ STEAP3 } & mRNA & Decreased & (45) \\
\hline & Protein & & \\
\hline \multirow[t]{2}{*}{ STEAP4 } & mRNA & Increased & (46) \\
\hline & Protein & & \\
\hline
\end{tabular}



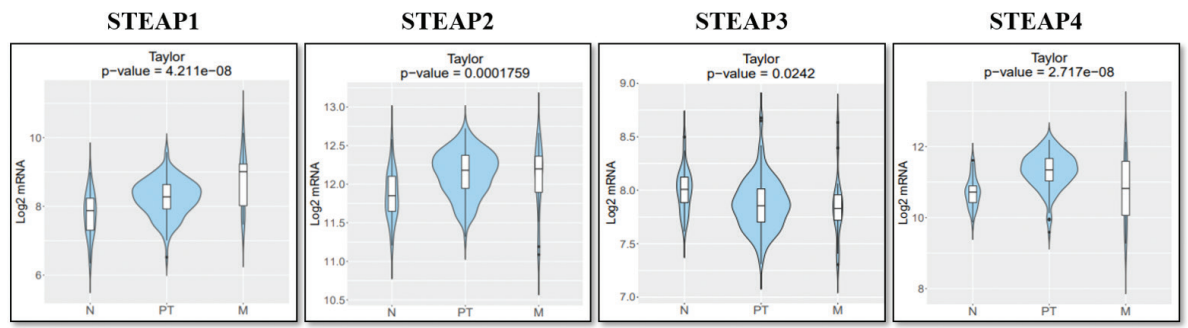

Figure 2. STEAPs expression with the progression of human prostate cancer using CANCERTOOL software. Violin plots show the expression of STEAPs in non-tumoral tissue (N), primary tumours (PT) and metastatic prostate cancer (M) specimens for Taylor dataset (51). This dataset has a cohort of 179 patients and is the only one with statistical significance for all STEAPs. However, the same results are verified in other dataset: for STEAP1, Grasso dataset (52); for STEAP2, Grasso (52), Tomlins (53) and Varambally (54) datasets; for STEAP4, Grasso (52) and Lapointe (55) dataset. Mean gene expression between the three groups was compared with an ANOVA test.

Regarding STEAP2, its expression levels were significantly higher in prostate carcinoma (Table 1). Several other studies have demonstrated the overexpression of STEAP2 in prostate cancer $(31,34,35)$. Burnell et al. showed that STEAP2 overexpression is positively correlated with the Gleason score (7). From the cBioPortal, the Prostate Adenocarcinoma dataset (51) showed that STEAP2 overexpression occurred in $16 \%$ of cases, while underexpressed in $1.6 \%$ of the cases. CANCERTOOL analysis also indicated a significantly higher expression of STEAP2 considering the Taylor dataset (Figure 2).

In contrast to other STEAPs, Porkka et al. described that STEAP3 expression decreases with the onset of prostate malignancy, suggesting that this gene may acts as a prostate tumor suppressor (Table 1) (46). Public datasets are in agreement with this study. Analysis of the Prostate Adenocarcinoma dataset (51) showed a downregulation of STEAP3 in 18\% of samples, whereas only $4 \%$ showed an overexpression. Also, CANCERTOOL software revealed a significantly diminished expression of STEAP3 in primary tumors and metastatic prostate cancer (Figure 2). However, Burnell et al. showed that there was no correlation between STEAP3 staining intensity and Gleason score (7). On the other hand, CANCERTOOL analysis indicated downregulation of STEAP3, and the low levels of STEAP3 are statistically correlated with high Gleason score.

STEAP4 subcellular localization is similar to that of STEAP2, and its expression also increases during prostate cancer development compared with nonmalignant prostate tissue (Table 1) (46). Analysis of the Prostate Adenocarcinoma dataset (51) retrieved from cBioPortal showed an increased expression of STEAP4 in $37.3 \%$ of samples, and a decreased expression in $4.7 \%$ the of the samples. Using the CANCERTOOL software, it was demonstrated that the increased STEAP4 expression correlated with prostate malignancy and the development of metastatic prostate cancer (Figure 2). Similar to STEAP3, no correlation between STEAP4 overexpression and Gleason score was observed (7), however, CANCERTOOL analysis showed that overexpression of STEAP4 is positively associated with Gleason grade. 


\section{PROGNOSTIC VALUE OF STEAP PROTEINS IN PROSTATE CARCINOMA}

STEAP family members are relatively new proteins and their potential as prognostic biomarkers have been demonstrated in breast cancer (56), glioblastoma (57, 58), Ewing tumors (30), skin disorders (59) and prostate cancer (7). A recent study that explored the use of STEAP proteins as possible prognostic indicators in prostate cancer showed that only STEAP4 was overexpressed, and significantly associated with relapse (7). To date, there are no studies evaluating the combined expression of different STEAP proteins. Thus, our intention in this chapter is to explore the association of STEAPs with prognosis of prostate cancer. A possible linear association between two variables, in this case, two genes, is analyzed by correlation coefficient (60), where - 1 and 1 indicate a negative and positive perfect linear relationship, respectively. Using primary prostate tumor tissue dataset from online MERAV database (Metabolic gEne Rapid Visualizer, http://merav.wi.mit.edu/ [accessed on December 2020]) a strong positive correlation was found between STEAP1 and STEAP2 expression (Table 2). This observation is in accordance with data of Grunewald et al., who noted that STEAP1 and STEAP2 seem to be significantly co-overexpressed across 59 cancer cell line entities (9), which suggests that STEAP2 is a likely candidate for heterodimerization with STEAP1. However, it is unknown if the combined expression of these two genes correlates with the overall survival of prostate cancer patients.

Using the same dataset retrieved from the cBioPortal (51), it was found that only STEAP1 overexpression is associated with the overall survival of prostate cancer patients (Figure 3A and Table 3). High expression of STEAP1 is associated with a shorter survival time, indicating a poor outcome. Concerning STEAP2, STEAP3 and STEAP4, no significant relationship was found between higher or low expression levels and the survival of prostate cancer patients (Table 3). These findings contradict the study of Burnell et al. (7) that reported a statistically significant difference between STEAP4 overexpression and overall survival, indicating that patients with higher STEAP4 levels were more likely to relapse earlier than those with medium or low expression levels (7). The source of the data, the number of patients per group, and the stratification of STEAPs expression may have caused the differences observed.

\begin{tabular}{lcccc}
\hline TABLE 2 & \multicolumn{4}{l}{$\begin{array}{l}\text { Gene correlation between of the STEAP family } \\
\text { members }\end{array}$} \\
& STEAP1 & STEAP2 & STEAP3 & STEAP4 \\
\hline STEAP1 & 1 & 0.9 & -0.09 & 0.43 \\
STEAP2 & 0.9 & 1 & -0.13 & 0.43 \\
STEAP3 & -0.09 & -0.13 & 1 & -0.03 \\
STEAP4 & 0.43 & 0.43 & -0.03 & 1 \\
\hline
\end{tabular}



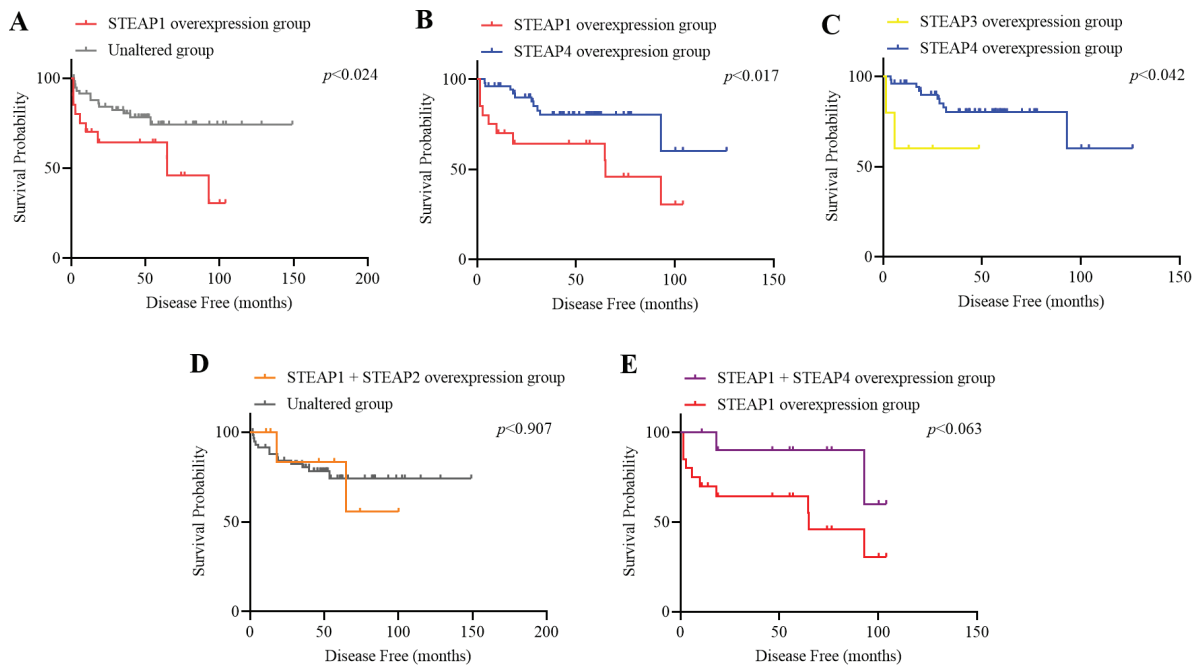

Figure 3. Expression of STEAP proteins and overall survival in prostate cancer patients. The Prostate Adenocarcinoma dataset (51), retrieved from cBioPoral, was stratified into groups with unaltered or overexpression of STEAPs, and different combinations were analyzed for overall survival. A. STEAP1 overexpression $(n=20)$ versus unaltered expression $(n=58)$ $(p<0.024)$. B. STEAP1 overexpression $(n=20)$ versus STEAP4 overexpression $(n=52)(p<0.017)$. C. STEAP3 overexpression $(n=5)$ versus STEAP4 overexpression $(n=52)(p<0.042)$. D. STEAP1 overexpression plus STEAP2 overexpression $(n=8)$ versus unaltered expression $(n=58)$ $(p<0.907)$. E. STEAP1 overexpression plus STEAP4 overexpression $(n=11)$ versus STEAP1 overexpression $(n=20)(p<0.063)$. Survival curves plotting fractional survival as a function of time was obtained using GraphPad Prisma 8.0.1.

As shown in Table 3 and considering all the possible associations between different STEAP proteins, patients with STEAPl overexpression displayed significantly lower overall survival compared with patients with overexpression of STEAP4 (Figure 3B). Furthermore, patients with STEAP3 overexpression presented poor survival outcome when compared with patients overexpressing STEAP4 (Figure 3C). These two associations with statistical significance suggest that the overexpression of STEAP4 can be a predictor for patients with prostate cancer since they presented a better survival rate when compared with those overexpressing STEAP1 and/or STEAP3. Additionally, a strong trend for better survival was observed in prostate cancer patients that presented both high expression of STEAP1 and STEAP4 compared with the ones that only overexpress STEAP1 (Figure 3E). The overexpression of both STEAP1 and STEAP2 could mean a potential good prediction for prostate cancer patients (Figure 3D), since the combined expression of high levels of STEAP1 and high levels of STEAP2 presented a better survival rate when compared with samples overexpressing STEAP1 only (Figure 3A). Despite the study limitations, such as the low number of patients in some experimental groups, these findings provide a comprehensive analysis of the STEAP expression in prostate cancer and their application for predicting prognosis of prostate cancer. 


\section{TABLE 3}

\section{Expression of STEAP family members correlated with overall survival of prostate cancer patients}

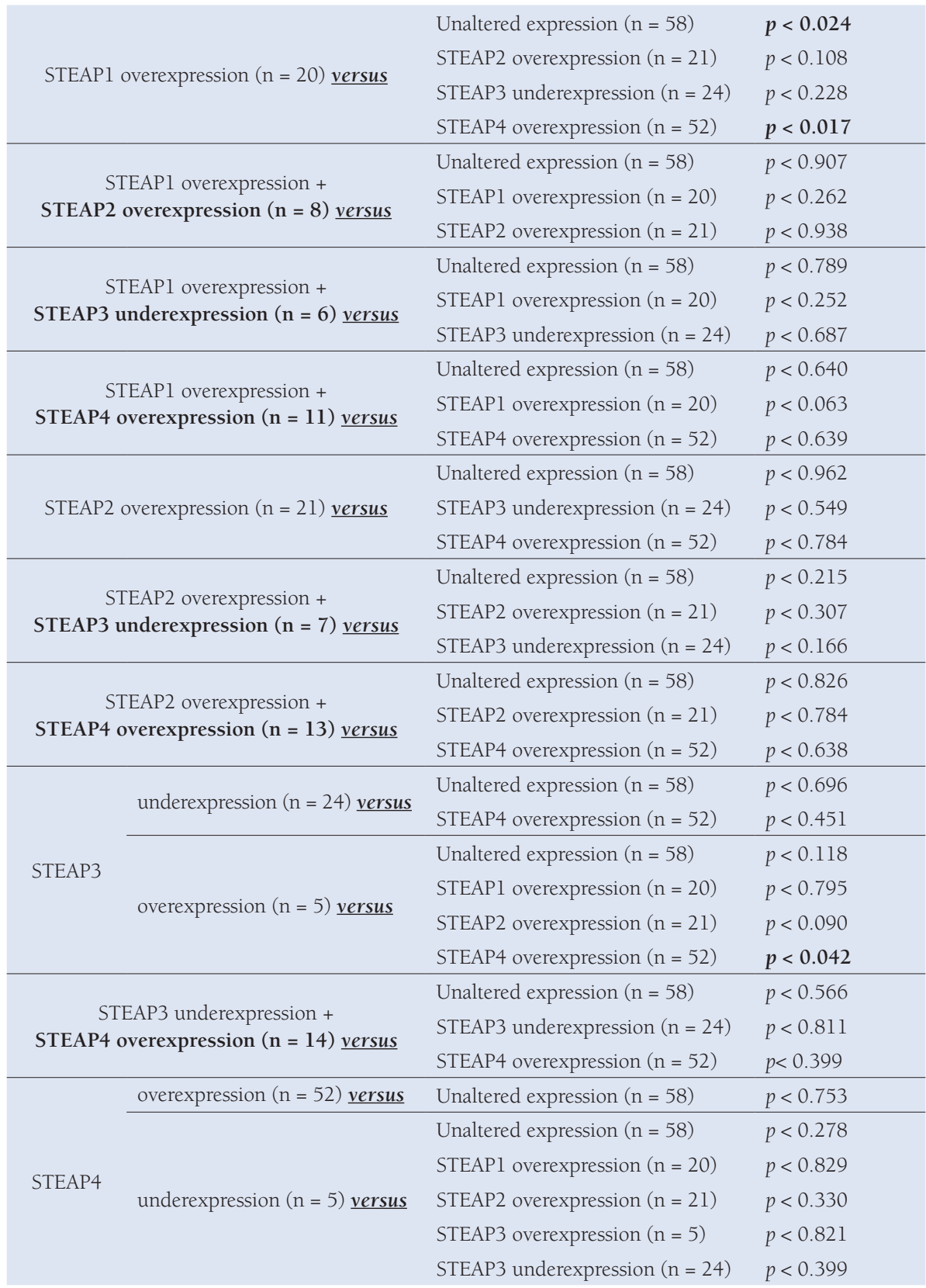

Data was extracted from Prostate Adenocarcinoma (MSKCC, 2010) (51) of cBioPortal and statistically analyzed using the GraphPad Prisma 8.0.1 software. Statistically significant differences considered for $p$-values $<0.05$ are highlighted in bold. 


\section{CONCLUSION}

Analyzing available scientific literature and multiple public databases show that STEAP1, STEAP2, and STEAP4 are overexpressed in prostate tumors. In contrast, STEAP3 is underexpressed. The differential expressions of these proteins appear to be of prognostic value. Overexpression of STEAPl overexpression is associated with poor clinical outcomes, whereas STEAP4 offers better overall survival and progression-free survival. However, further investigations in large scale clinical cohorts are needed to definitively confirm the prognostic value of the STEAPs proteins, and the therapeutic potential of targeting STEAPs for prostate cancer.

Acknowledgement: The authors acknowledge the support from FEDER funds through the POCI - COMPETE 2020 - Operational Programme Competitiveness and Internationalisation in Axis I - Strengthening research, technological development and innovation (Project No. 007491; Project No 029114), National Funds by FCT-Foundation for Science and Technology (Project UID/Multi/00709/2019) and Applied Molecular Biosciences Unit - UCIBIO which is financed by national funds from FCT (UIDP/04378/2020 and UIDB/04378/2020). Sandra M Rocha and Jorge Barroca-Ferreira were funded by $\mathrm{PhD}$ Fellowship (SFRH/ BD/115693/2016 and SFRH/BD/130068/2017, respectively) and Luís A. Passarinha by sabbatical fellowship (SFRH/BSAB/150376/2019) from FCT.

Conflict of interest: The authors declare no potential conflicts of interest with respect to research, authorship and/or publication of this manuscript.

Copyright and permission statement: The authors confirm that the materials included in this chapter do not violate copyright laws. Where relevant, appropriate permissions have been obtained from the original copyright holder(s), and all original sources have been appropriately acknowledged or referenced.

\section{REFERENCES}

1. Culp MBB, Soerjomataram I, Efstathiou JA, Bray F, Jemal A. Recent Global Patterns in Prostate Cancer Incidence and Mortality Rates. Vol. 77, European Urology. Elsevier B.V.; 2020. p. 38-52. https://doi. org/10.1016/j.eururo.2019.08.005

2. Rawla P. Epidemiology of Prostate Cancer. World J Oncol. 2019;10(2):63-89. https://doi. org/10.14740/wjon1191

3. Cary KC, Cooperberg MR. Biomarkers in prostate cancer surveillance and screening: Past, present, and future. Vol. 5, Therapeutic Advances in Urology. SAGE Publications; 2013. p. 318-29. https:// doi.org/10.1177/1756287213495915

4. Gomes IM, Rocha SM, Gaspar C, Alvelos MI, Santos CR, Socorro S, et al. Knockdown of STEAP1 inhibits cell growth and induces apoptosis in LNCaP prostate cancer cells counteracting the effect of androgens. Med Oncol. 2018;35(3). https://doi.org/10.1007/s12032-018-1100-0

5. Grunewald TGP, Diebold I, Esposito I, Plehm S, Hauer K, Thiel U, et al. STEAPI is associated with the invasive and oxidative stress phenotype of ewing tumors. Mol Cancer Res. 2012 Jan;10(1):52-65. https://doi.org/10.1158/1541-7786.MCR-11-0524

6. Ohgami RS, Campagna DR, McDonald A, Fleming MD. The Steap proteins are metalloreductases. Blood. 2006;108(4):1388-94. https://doi.org/10.1182/blood-2006-02-003681 
7. Burnell SEA, Spencer-Harty S, Howarth S, Bodger O, Kynaston H, Morgan C, et al. Utilisation of the STEAP protein family in a diagnostic setting may provide a more comprehensive prognosis of prostate cancer. PLoS One. 2019;14(8):e0220456. https://doi.org/10.1371/journal.pone.0220456

8. Gomes IM, Maia CJ, Santos CR. STEAP proteins: From structure to applications in cancer therapy. Vol. 10, Mol Cancer Res; 2012. p. 573-87. https://doi.org/10.1158/1541-7786.MCR-11-0281

9. Grunewald TGP, Bach H, Cossarizza A, Matsumoto I. The STEAP protein family: Versatile oxidoreductases and targets for cancer immunotherapy with overlapping and distinct cellular functions. Biol Cell. 2012;104(11):641-57. https://doi.org/10.1111/boc.201200027

10. Moreaux J, Kassambara A, Hose D, Klein B. STEAPl is overexpressed in cancers: A promising therapeutic target. Biochem Biophys Res Commun. 2012;429(3-4):148-55. https://doi.org/10.1016/j. bbrc.2012.10.123

11. Barroca-Ferreira J, Pais JP, Santos MM, Goncalves AM, Gomes IM, Sousa I, et al. Targeting STEAP1 Protein in Human Cancer: Current Trends and Future Challenges. Curr Cancer Drug Targets. 2017;18(3):222-30. https://doi.org/10.2174/1568009617666170427103732

12. Cerami E, Gao J, Dogrusoz U, Gross BE, Sumer SO, Aksoy BA, et al. The cBio Cancer Genomics Portal: An open platform for exploring multidimensional cancer genomics data. Cancer Discov. 2012;2(5):401-4. https://doi.org/10.1158/2159-8290.CD-12-0095

13. Cortazar AR, Torrano V, Martín-Martín N, Caro-Maldonado A, Camacho L, Hermanova I, et al. Cancertool: A visualization and representation interface to exploit cancer datasets. Cancer Res. 2018;78(21):6320-8. https://doi.org/10.1158/0008-5472.CAN-18-1669

14. Kleven MD, Dlakić M, Lawrence CM. Characterization of a single b-type heme, FAD, and metal binding sites in the transmembrane domain of sixtransmembrane epithelial antigen of the prostate (STEAP) family proteins. J Biol Chem. 2015;290(37):22558-69. https://doi.org/10.1074/jbc.M115.664565

15. Oosterheert W, Reis J, Gros P, Mattevi A. An elegant four-helical fold in NOX and STEAP enzymes facilitates electron transport across biomembranes - Similar vehicle, different destination. Acc Chem Res. 2020;53(9):1969-80. https://doi.org/10.1021/acs.accounts.0c00400

16. Hubert RS, Vivanco I, Chen E, Rastegar S, Leong K, Mitchell SC, et al. STEAP: A prostate-specific cell-surface antigen highly expressed in human prostate tumors. Proc Natl Acad Sci U S A. 1999;96(25):14523-8. https://doi.org/10.1073/pnas.96.25.14523

17. Knutson MD. Steap Proteins: Implications for Iron and Copper Metabolism. Nutr Rev. 2008;65(7): 335-40. https://doi.org/10.1111/j.1753-4887.2007.tb00311.x

18. Kim K, Mitra S, Wu G, Berka V, Song J, Yu Y, et al. Six-Transmembrane Epithelial Antigen of Prostate 1 (STEAP1) Has a Single b Heme and Is Capable of Reducing Metal Ion Complexes and Oxygen. Biochemistry. 2016;55(48):6673-84. https://doi.org/10.1021/acs.biochem.6b00610

19. Yamamoto T, Tamura Y, Kobayashi J ichi, Kamiguchi K, Hirohashi Y, Miyazaki A, et al. Sixtransmembrane epithelial antigen of the prostate-1 plays a role for in vivo tumor growth via intercellular communication. Exp Cell Res. 2013;319(17):2617-26. https://doi.org/10.1016/j.yexcr.2013.07.025

20. Esmaeili S-A, Nejatollahi F, Sahebkar A. Inhibition of Intercellular Communication between Prostate Cancer Cells by A Specific Anti-STEAP-1 Single Chain Antibody. Anticancer Agents Med Chem. 2017;18(12):1674-9. https://doi.org/10.2174/1871520618666171208092115

21. Oosterheert W, Gros P. Cryo-electron microscopy structure and potential enzymatic function of human six-transmembrane epithelial antigen of the prostate 1 (STEAP1). J Biol Chem. 2020;295(28): 9502-12. https://doi.org/10.1074/jbc.RA120.013690

22. Oosterheert W, van Bezouwen LS, Rodenburg RNP, Granneman J, Förster F, Mattevi A, et al. Cryo-EM structures of human STEAP4 reveal mechanism of iron(III) reduction. Nat Commun. 2018;9(1). https://doi.org/10.1038/s41467-018-06817-7

23. Schober SJ, Thiede M, Gassmann H, Prexler C, Xue B, Schirmer D, et al. MHC Class I-Restricted TCRTransgenic CD4+ T Cells Against STEAP1 Mediate Local Tumor Control of Ewing Sarcoma In Vivo. Cells. 2020;9(7):1581. https://doi.org/10.3390/cells9071581

24. Danila DC, Szmulewitz RZ, Vaishampayan U, Higano CS, Baron AD, Gilbert HN, et al. Phase i Study of DSTP3086S, an antibody-drug conjugate targeting six-transmembrane epithelial antigen of prostate 1, in metastatic castration-resistant prostate cancer. J Clin Oncol. 2019;37(36):3518-27. https:// doi.org/10.1200/JCO.19.00646 
25. Carrasquillo JA, Fine BM, Pandit-Taskar N, Larson SM, Fleming SE, Fox JJ, et al. Imaging patients with metastatic castration-resistant prostate cancer using 89Zr-DFO-MSTP2109A anti-STEAP1 antibody. J Nucl Med. 2019;60(11):1517-23. https://doi.org/10.2967/jnumed.118.222844

26. Jiao Z, Huang L, Sun J, Xie J, Wang T, Yin X, et al. Six-transmembrane epithelial antigen of the prostate 1 expression promotes ovarian cancer metastasis by aiding progression of epithelial-to-mesenchymal transition. Histochem Cell Biol. 2020. https://doi.org/10.1007/s00418-020-01877-7

27. Ihlaseh-Catalano SM, Drigo SA, de Jesus CMN, Domingues MAC, Trindade Filho JCS, de Camargo JL V., et al. STEAP1 protein overexpression is an independent marker for biochemical recurrence in prostate carcinoma. Histopathology. 2013;63(5):678-85. https://doi.org/10.1111/his. 12226

28. Gomes IM, Arinto P, Lopes C, Santos CR, Maia CJ. STEAPl is overexpressed in prostate cancer and prostatic intraepithelial neoplasia lesions, and it is positively associated with Gleason score. Urol Oncol Semin Orig Investig. 2014;32(1):53.e23-53.e29. https://doi.org/10.1016/j.urolonc.2013.08.028

29. Yu-zhuo Pan, Yang Li, Li-rong Guo, Yan-ying Zhao XZ. Influence of expression of six transmembrane epithelial antigen of the prostate-1 on intracellular reactive oxygen species level and cell growth: an in vitro experiment. Zhonghua Yi Xue Za Zhi . 2008;88(9):64l-4.

30. Cheung IY, Feng Y, Danis K, Shukla N, Meyers P, Ladanyi M, et al. Novel markers of subclinical disease for Ewing family tumors from gene expression profiling. Clin Cancer Res. 2007;13(23):6978-83. https://doi.org/10.1158/1078-0432.CCR-07-1417

31. Korkmaz KS, Elbi C, Korkmaz CG, Loda M, Hager GL, Saatcioglu F. Molecular cloning and characterization of STAMP1, a highly prostate-specific six transmembrane protein that is overexpressed in prostate cancer. J Biol Chem. 2002;277(39):36689-96. https://doi.org/10.1074/jbc. M202414200

32. Porkka KP, Helenius MA, Visakorpi T. Cloning and characterization of a novel six-transmembrane protein STEAP2, expressed in normal and malignant prostate. Lab Investig. 2002;82(11):1573-82. https://doi.org/10.1097/01.LAB.0000038554.26102.C6

33. Wang L, Jin Y, Arnoldussen YJ, Jonson I, Qu S, Mælandsmo GM, et al. STAMP1 is both a proliferative and an antiapoptotic factor in prostate cancer. Cancer Res. 2010;70(14):5818-28. https://doi. org/10.1158/0008-5472.CAN-09-4697

34. Whiteland H, Spencer-Harty S, Morgan C, Kynaston H, Thomas DH, Bose P, et al. A role for STEAP2 in prostate cancer progression. Clin Exp Metastasis. 2014;31(8):909-20. https://doi.org/10.1007/ s10585-014-9679-9

35. Burnell SEA, Spencer-Harty S, Howarth S, Bodger O, Kynaston H, Morgan C, et al. STEAP2 Knockdown Reduces the Invasive Potential of Prostate Cancer Cells. Sci Rep. 2018 Dec 1;8(1). https:// doi.org/10.1038/s41598-018-24655-x

36. Ohgami RS, Campagna DR, Greer EL, Antiochos B, McDonald A, Chen J, et al. Identification of a ferrireductase required for efficient transferrin-dependent iron uptake in erythroid cells. Nat Genet. 2005;37(11):1264-9. https://doi.org/10.1038/ng1658

37. Sendamarai AK, Ohgami RS, Fleming MD, Lawrence CM. Structure of the membrane proximal oxidoreductase domain of human Steap3, the dominant ferrireductase of the erythroid transferrin cycle. Proc Natl Acad Sci U S A. 2008;105(21):7410-5. https://doi.org/10.1073/pnas.0801318105

38. Lespagnol A, Duflaut D, Beekman C, Blanc L, Fiucci G, Marine JC, et al. Exosome secretion, including the DNA damage-induced p53-dependent secretory pathway, is severely compromised in TSAP6/Steap3-null mice. Cell Death Differ. 2008;15(11):1723-33. https://doi.org/10.1038/ cdd.2008.104

39. Wan C, Fu J, Wang Y, Miao S, Song W, Wang L. Exosome-related multi-pass transmembrane protein TSAP6 is a target of rhomboid protease RHBDDl-induced proteolysis. PLoS One. 2012;7(5). https:// doi.org/10.1371/journal.pone.0037452

40. Wilkinson HN, Upson SE, Banyard KL, Knight R, Mace KA, Hardman MJ. Reduced Iron in Diabetic Wounds: An Oxidative Stress-Dependent Role for STEAP3 in Extracellular Matrix Deposition and Remodeling. J Invest Dermatol. 2019;139(11):2368-2377.e7. https://doi.org/10.1016/j. jid.2019.05.014

41. Zhang F, Tao Y, Zhang Z, Guo X, An P, Shen Y, et al. Metalloreductase steap3 coordinates the regulation of iron homeostasis and inflammatory responses. Haematologica. 2012;97(12):1826-35. https://doi. org/10.3324/haematol.2012.063974 
42. Feng Z. p53 regulation of the IGF-1/AKT/mTOR pathways and the endosomal compartment. [Internet]. Vol. 2, Cold Spring Harbor perspectives in biology. Cold Spring Harb Perspect Biol; 2010. https://doi.org/10.1101/cshperspect.a001057

43. Zhang X, Steiner MS, Rinaldy A, Lu Y. Apoptosis induction in prostate cancer cells by a novel gene product, pHyde, involves caspase-3. Oncogene. 2001;20(42):5982-90. https://doi.org/10.1038/ sj.onc. 1204831

44. M S Steiner, X Zhang, Y Wang YL. Growth inhibition of prostate cancer by an adenovirus expressing a novel tumor suppressor gene, pHyde. Cancer Res . 2000;60(16):4419-25.

45. Porkka KP, Nupponen NN, Tammela TLJ, Vessella RL, Visakorpi T. Human pHyde is not a classical tumor suppressor gene in prostate cancer. Int J Cancer. 2003;106(5):729-35. https://doi.org/10.1002/ ijc. 11278

46. Korkmaz CG, Korkmaz KS, Kurys P, Elbi C, Wang L, Klokk TI, et al. Molecular cloning and characterization of STAMP2, an androgen-regulated six transmembrane protein that is overexpressed in prostate cancer. Oncogene. 2005;24(31):4934-45. https://doi.org/10.1038/sj.onc.1208677

47. Scarl RT, Lawrence CM, Gordon HM, Nunemaker CS. STEAP4: Its emerging role in metabolism and homeostasis of cellular iron and copper. Vol. 234, Journal of Endocrinology. 2017. p. R123-34. https://doi.org/10.1530/JOE-16-0594

48. Jin Y, Wang L, Qu S, Sheng X, Kristian A, Mælandsmo GM, et al. STAMP 2 increases oxidative stress and is critical for prostate cancer . EMBO Mol Med. 2015;7(3):315-31. https://doi.org/10.15252/ emmm.201404181

49. Tamura T, Chiba J. STEAP4 regulates focal adhesion kinase activation and CPG motifs within STEAP4 promoter region are frequently methylatedin DU145, human androgen-independent prostate cancer cells. Int J Mol Med. 2009;24(5):599-604. https://doi.org/10.3892/ijmm_00000270

50. Wang Q, Tiffen J, Bailey CG, Lehman ML, Ritchie W, Fazli L, et al. Targeting amino acid transport in metastatic castration-resistant prostate cancer: Effects on cell cycle, cell growth, and tumor development. J Natl Cancer Inst. 2013;105(19):1463-73. https://doi.org/10.1093/jnci/djt241

51. Taylor BS, Schultz N, Hieronymus H, Gopalan A, Xiao Y, Carver BS, et al. Integrative Genomic Profiling of Human Prostate Cancer. Cancer Cell. 2010;18(1):11-22. https://doi.org/10.1016/j. ccr.2010.05.026

52. Grasso CS, Wu YM, Robinson DR, Cao X, Dhanasekaran SM, Khan AP, et al. The mutational landscape of lethal castration-resistant prostate cancer. Nature. 2012;487(7406):239-43. https://doi. org/10.1038/nature 11125

53. Tomlins SA, Mehra R, Rhodes DR, Cao X, Wang L, Dhanasekaran SM, et al. Integrative molecular concept modeling of prostate cancer progression. Nat Genet. 2007;39(1):41-51. https://doi.org/10.1038/ ng1935

54. Varambally S, Yu J, Laxman B, Rhodes DR, Mehra R, Tomlins SA, et al. Integrative genomic and proteomic analysis of prostate cancer reveals signatures of metastatic progression. Cancer Cell. 2005;8(5):393-406. https://doi.org/10.1016/j.ccr.2005.10.001

55. Lapointe J, Li C, Higgins JP, Van De Rijn M, Bair E, Montgomery K, et al. Gene expression profiling identifies clinically relevant subtypes of prostate cancer. Proc Natl Acad Sci U S A. 2004;101(3): 811-6. https://doi.org/10.1073/pnas.0304146101

56. Wu HT, Chen WJ, Xu Y, Shen JX, Chen WT, Liu J. The Tumor Suppressive Roles and Prognostic Values of STEAP Family Members in Breast Cancer. Biomed Res Int. 2020. https://doi. org/10.1155/2020/9578484

57. Chen H, Xu C, Yu Q, Zhong C, Peng Y, Chen J, et al. Comprehensive landscape of STEAP family functions and prognostic prediction value in glioblastoma. J Cell Physiol. 2020;jcp.30060. https://doi. org/10.1002/jcp.30060

58. Prasad B, Tian Y, Li X. Large-Scale Analysis Reveals Gene Signature for Survival Prediction in Primary Glioblastoma. Mol Neurobiol. 2020;57(12):5235-46. https://doi.org/10.1007/s12035-020-02088-w

59. Liang Y, Xing X, Beamer MA, Swindell WR, Sarkar MK, Roberts LW, et al. Six-transmembrane epithelial antigens of the prostate comprise a novel inflammatory nexus in patients with pustular skin disorders. J Allergy Clin Immunol. 2017;139(4):1217-27. https://doi.org/10.1016/j.jaci.2016.10.021

60. Nyirongo VB, Mukaka MM, Kalilani-Phiri L V. Statistical pitfalls in medical research. Malawi Med J. 2008;20(1). https://doi.org/10.4314/mmj.v20il.10949 
